\title{
Análisis cienciométrico de las publicaciones sobre orientación, tutoría y acción tutorial registradas en bases de datos
}

\author{
Jorge Expósito-López* y Eva María Olmedo-Moreno \\ Universidad de Granada, Facultad de Ciencias de la Educación, Dpto. MIDE, Campus de Cartuja, 18071, Granada- \\ España. (correo-e: jorgeel@ugr.es; emolmedo@ugr.es)
}

* Autor a quien debe ser dirigida la correspondencia.

Recibido Nov. 15, 2019; Aceptado Ene. 13, 2020; Versión final Ene. 27, 2020, Publicado Jun. 2020

\begin{abstract}
Resumen
Este estudio tiene como finalidad general mejorar la comprensión y descripción del campo de la orientación, tutoría y acción tutorial mediante un análisis cienciométrico. Se recurre a una metodología de registro y análisis mixto (SPSSStatistics y Nvivo ) de la producción de 1054 artículos de investigación registrados en las bases de datos SCOPUS, ISOC, ProQuest y Redinet. Los resultados muestran patrones no esperados de crecimiento de la productividad y autoría personal e institucional. Se muestran limitaciones referidas al acceso y recuperación de documentos para su examen y registro adecuado que se superan con el uso de diversas bases de datos y una muestra amplia. Se puede concluir que la investigación en esta disciplina emergente posee madurez productiva y una adecuación a los nuevos y actuales tópicos educativos como atención a la diversidad o desarrollo personal. Aunque requiere de estudios de revisión que permitan obtener una visión del campo de conocimiento.
\end{abstract}

Palabras clave: orientación; tutoría; acción tutorial; cienciometría; investigación

\section{Scientometric analysis of publications on guidance, mentoring, and tutorial action registered on databases}

\begin{abstract}
This study aims to understand and describe the field of guidance, tutoring, and tutorial action by performing a scientometric analysis of 1054 research articles. The articles were retrieved from four databases (SCOPUS, ISOC, ProQuest and Redinet) and were examined to record and analyze (SPSSStatistics and Nvivo) the production of academic research articles. The results showed an unexpected pattern of productivity in personal and institutional authorship. There were also limitations related to access and retrieval of the research articles for their examination and proper registration. But, these limitations were overcomed by using multiple databases and a large sample size. It can be concluded that research on guidance, mentoring, and tutorial action has achieved research production maturity and has adaptated succesfully to new and current educational topics such as attention to diversity and personal development. However, the field still requires review studies that allow obtaining a vision of its field of knowledge.
\end{abstract}




\section{INTRODUCCIÓN}

Los trabajos Solla Price y Garfield establecen, con la creación de la base de datos Science Citation Index (SCI) en 1963 publicado por el Institute of Scientific Information (ISI), el inicio de los estudios cienciométricos en la medición de la producción científica y su impacto (Callon, Courtial y Penan, 1995). Siendo en la actualidad un campo afianzado y con alto crecimiento, con utilidad para la propia ciencia al analizar sus producciones, y mejorar la comprensión y descripción del campo científico mediante estudios sistemáticos de síntesis y análisis consistentes y fiables.

La evaluación de la investigación, de la que forman parte los estudios cienciométricos, se entiende como una necesidad indispensable para el mundo científico (Sánchez-Ruíz, 2005) y una condición imprescindible para el avance de la propia ciencia (Bracho, Torralbo, Maz-Machado y Adamuz, 2014). No sólo porque permite comprender mejor la realidad, sino porque facilita una visión más clara para transformarla como parte de su responsabilidad social.

De esta forma los estudios cienciométricos responden al análisis de la cantidad y calidad de los trabajos científicos mediante el uso de diversos indicadores, por lo que se han convertido en un elemento transversal a la propia ciencia, como ya planteaban Spiegel-Rosing y Price (1977), que permiten su descripción cuantificada en un corpus de conocimiento perfectamente organizado (Van Raan y Tijssen,1990 y Leydesdorff, 2001), con una indudable la influencia de Merton (1965) que incluso lo asociaba a la propia construcción social. Y estos indicadores han pasado de una perspectiva bibliométrica, considerando niveles de productividad e identificando autoría personal, institucional o fuentes de publicación, entre otros; para adoptar una perspectiva cienciométrica, que con implicaciones más profundas, incorporan análisis de contenido de los títulos y palabras clave, elementos sobre metodologías e instrumentos empleados o incluso posibles consideraciones sobre la efectividad de las investigaciones analizadas (Brooks, 1986).

La orientación, la tutorización y más recientemente la acción tutorial, constituyen un elemento fundamental de los procesos de transformación de la educación actual debido a su ajuste a las transformaciones sociales, nuevos contextos y herramientas de aprendizaje o diversidad de usuarios de los sistemas de formación. Por lo que la utilidad de los estudios cienciométricos en esta disciplina serían un elemento importante de reflexión y toma de decisiones fundamentada sobre los cambios educativos para la mejora. Sin embargo, éstos se limitan en la mayoría de los casos a estudios centrados en la productividad de una revista científica concreta (López-Gómez, 2016) o de investigaciones originadas en tesis doctorales registras en una única base de datos como TESEO (Ferreira-Villa, Pascual-García, Pol-Asmarats; 2013 o Salgado-Orellana, Ferrada, Puraivan y Parraguez, 2018) que solo ofrece informaciones a modo de resumen. Y que por tanto ofrecen un estudio sesgado y una visión disciplinar limitada.

En este sentido, tanto por la importancia que sus practicas está cobrando en el desarrollo actual como por su utilidad en los procesos de transformación y mejora educativa, se requieren estudios cienciométricos que permitan ofrecer desde esta perspectiva evaluativa una visión global de la disciplina, considerando tipos de trabajos, fuentes de publicación y bases de datos de registro diversas. Siendo éste el origen del planteamiento del tópico o problema de investigación "cómo es la evolución de la producción científica sobre orientación, tutoría y acción tutorial" y del objetivo general del estudio (OG) "realizar un análisis cienciométrico de la producción de artículos de investigación académica sobre orientación, tutoría y acción tutorial registrada en bases de datos."

Este objetivo general se concreta en objetivos específicos (OE) modelados por los indicadores y variables de estudio que pueden tratarse en esta tipología de investigaciones (Fernández-Cano y Bueno, 1999 o Expósito, 2014), de los que se han considerado en este trabajo sobre la investigación académica sobre orientación, tutoría y acción tutorial registrada en bases de datos como artículos científicos: $\left(\mathrm{OE}_{1}\right)$ Analizar la producción para describir su desarrollo diacrónico y patrones de crecimiento; $\left(\mathrm{OE}_{2}\right)$ Analizar la productividad personal e institucional y productividad por género de los autores; $\left(\mathrm{OE}_{3}\right)$ Comprobar los contenidos usuales de esta tipología de trabajos a través del análisis cualitativo de las palabras contenidas en sus títulos y de sus palabras clave. Analizando la evolución de la complejidad disciplinar desde la evolución cuantitativa de la longitud de sus títulos; $\left(\mathrm{OE}_{4}\right)$ Analizar la publicación de los trabajos en cuanto a la identificación nominal de la fuente empleada y su tipología, la lengua de redacción y el país en el que se publica; (OE5) Analizar la citación en cuanto a la evolución de las citas realizadas, identificación de los autores más citados, y el número de citas recibidos por los trabajos objeto de análisis; y $\left(\mathrm{OE}_{6}\right)$ Analizar los aspectos metodológicos usuales en cuanto a la metodología empleada e instrumentos más usuales para la recogida de información.

\section{METODOLOGÍA}

La metodología es de carácter descriptivo mediante un estudio de revisión documental. Y la población objeto de estudio sería la investigación sobre el tópico de investigación registrada en bases de datos: SCOPUS, ISOC, ProQuest and Redinet. Y el muestreo, aunque con intencionalidad censal, se limita de forma incidental en un proceso polietápico ajustado a las distintas fases de la investigación. En una fase preliminar, sobre 
aspectos previos de la investigación, se realizan búsquedas preliminares basadas en los hallazgos del marco teórico para establecer términos de búsqueda sobre la temática del trabajo. En la primera, fase se delimitan los indicadores y conectores boleanos por la utilidad de los resultados de búsqueda que facilitan y se toman decisiones sobre las bases de datos más útiles en función de resultados preliminares mediante acuerdo de los cinco jueces expertos participantes en esta fase. En una segunda fase se conforman los indicadores de búsqueda, basándose en el proceso previo de revisión de la teoría y los ensayos prácticos, y se construye la base de datos o instrumento de registro, que tiene validez de uso como mencionan Bueno (2002), Build, Beltrán, Miedes y Martínez (2010) y Fernández-Bautista, Torralbo y Cano (2014). Y en la tercera fase se realiza el registro de los datos de la selección maestral que se refleja en la tabla 1.

Tabla 1: Registros localizados en la búsqueda en bases de datos

\begin{tabular}{|l|l|r|r|}
\hline \multirow{2}{*}{ Descriptores filtrados } & \multirow{2}{*}{$\begin{array}{l}\text { Bases de } \\
\text { datos }\end{array}$} & \multicolumn{2}{|c|}{ Resultados de la búsqueda } \\
\cline { 2 - 4 } & & Localizados & $\begin{array}{r}\text { Productores de } \\
\text { datos }\end{array}$ \\
\hline \multirow{3}{*}{$\begin{array}{l}\text { orientación, tutoría y tutor, } \\
\text { acción tutorial, guidance, } \\
\text { orientation, mentoring, tutoring. }\end{array}$} & Scopus & 892 & 307 \\
\cline { 2 - 4 } & ERIC & 455 & 102 \\
\cline { 2 - 4 } & Redinet & 2345 & 284 \\
\cline { 2 - 4 } & ProQuest & 11444 & 248 \\
\cline { 2 - 4 } & Otras & 286 & 11 \\
\hline & TOTAL & 15941 & 1054 \\
\hline
\end{tabular}

La muestra productora se ajusta a la posibilidad de recuperación del texto completo del artículo de investigación y gratuidad de la descarga del documento, que facilitara su revisión y registro de las variables consideradas en el estudio. Y posteriormente a su revisión para constatar el ajuste real del artículo al tópico de investigación como "trabajos sobre orientación, tutoría y acción tutorial educativa" y la calidad intrínseca del informe "estudios experimentales que hacen referencia en su informe a todas las fases de un proceso de investigación reglado, recogen y discuten datos y establecen conclusiones", de forma previa a su registro como muestra productora de datos.

\section{RESULTADOS}

El análisis de los resultados se realiza de forma ordenada atendiendo a los indicadores cienciométricos usuales en este tipo de estudios.

\section{Productividad diacrónica}

El análisis diacrónico de la productividad muestra una producción de solo 13 trabajos sobre esta temática en el periodo anterior a 1988, posiblemente debido a la falta de registros digitales de los trabajos, por lo que aunque se indica aquí a título informativo, no se considera dicho periodo en el análisis diacrónico porque dificultaría su interpretación. La productividad muestra, como figura en la tabla 2, un incremento decenal de $56 / 347 / 638$, con una tasa de incremento en este último decenio del $61,29 \%$ del total y una tasa de crecimiento de 27,95. Lo que supone la duplicación de la primera década considerada (fa=56). Y este patrón de crecimiento se ajusta a un modelo logístico recogido en la segunda ley de crecimiento de Price. este aumento de la productividad diacrónica, referida al análisis de la autoría, conlleva un aumento del número de autores (ver tabla 3$)$ en valores absolutos anuales y decenalmente (102/727/1565).

En esta tabla, la notación ( $\Sigma$.art/ $\Sigma$.aut) hace referencia al cociente entre el total de artículos publicados y el total de autores en el periodo indicado, I. nC. o \% no colaboración hace referencia al número de autores en solitario/artículos totales del periodo; y el I.C. o Índice de colaboración al número de autores en colaboración/artículos totales del periodo. Y muestra que el número total de autores que firman los trabajos de 1,82 autores, a 2,10 y hasta 2,45, lo que supone un aumento significativo de la colaboración en la autoría en la disciplina. Esto supone un índice de colaboración, representado por el cociente del total de documentos en coautoría y el total de documentos producido en un periodo que muestra un aumento diferencias del I.C. de 0,93 .

La productividad diacrónica en el tópico, referido a la investigación sobre orientación, tutorización y acción tutorial, muestra un incremento creciente ajustado a una curva logística, lo que supone el interés progresivo y cierta madurez productiva. $Y$ el incremento del número total absoluto de autores, pero también del número de autores y del índice de colaboración describe que s produce más, pero también se colabora más para 
hacerlo. Sin embargo, este índice sigue por debajo del número usual de autores de 3, mencionado en los criterios de evaluación de la CNEAI en la Resolución de 23 de noviembre de 2017, de la Comisión Nacional Evaluadora de la Actividad Investigadora, por lo que se publican los criterios específicos aprobados para cada uno de los campos de evaluación. En el campo 7. Ciencias Sociales, Políticas, del Comportamiento y de la Educación en el apartado 2; donde se menciona que "el número de autores de una aportación deberá estar justificado por el tema, su complejidad y su extensión” (MECD, 2017, p.117070).

Tabla 2: Índice de colaboración y productividad en periodos diacrónicos

\begin{tabular}{|c|c|c|c|c|c|c|}
\hline Periodo & 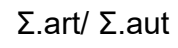 & Mono-autoría & Multi-autoría & № artículos & I. nC. & I.C. \\
\hline $1988 / 1998$ & 1,82 & 36 & 96 & 69 & 0,52 & 1,39 \\
\hline $1999 / 2008$ & 2,10 & 124 & 459 & 347 & 0,36 & 1,32 \\
\hline 2009/2019 & 2,45 & 211 & 1494 & 638 & 0,33 & 2,34 \\
\hline TOTAL & 2,30 & 371 & 2049 & 1054 & 0,35 & 1,94 \\
\hline
\end{tabular}

\section{Autoría nominal}

La autoría nominal personal se muestra en la tabla 3 por autores, número de autores y el número de aportaciones, que permite establecer el Índice de productividad personal, según la ISO 5127/3A (1981). Estos datos muestran un alto índice de transitoriedad, puesto que el 94,98 de los autores solo realizan una aportación, índice que Spinak (1996) fijaba en Ciencias Sociales en el 75\%. Es decir, existe un número limitado de autores especialistas, ajustado a las predicciones ajustadas en la Ley de Lotka, lo que supone una falta de especialización disciplinar o al menos una falta de investigación publicada sobre la materia, ya que en muchas ocasiones las prácticas tutoriales se han fundamentado más en la práctica docente y experiencias personales, que en la formación disciplinar.

El análisis del género de los autores se realiza por orden de ésta, así como de forma diacrónica. Y muestra un número de hombres menor que el de mujeres en todos los periodos considerados, así como de forma global $(\mathrm{H}=1034<\mathrm{M}=1346)$. Sin embargo, si atendemos al orden de autoría y consideramos el primer autor, como se muestra en la tabla 4, se aprecia un mayor número de hombres, como primeros autores, hasta la última década de 2009 a 2019 en la que se ajusta al porcentaje global con un mayor número de autoras. Esto corresponde aun patrón de publicación, sobre el que se ha avanzado significativamente en los últimos años, pero que todavía requiere de políticas de apoyo que fomenten la igualdad de género en la producción científica, como se muestra en los informes de la Secretaría de Estado de Universidades, Investigación y Desarrollo (2017) o de la Comisión Europea o European Commission (2014).

Tabla 3: Autoría nominal con más de diez aportaciones y número de autores por aportaciones

\begin{tabular}{|l|c|c|}
\hline Autores & $\mathrm{n}$ & $\mathrm{f}$ \\
\hline Bandura, A. & 1 & 56 \\
\hline Barrows, H. & 1 & 50 \\
\hline Bisquerra Alzina, R & 1 & 48 \\
\hline Dolmans, D. & 1 & 37 \\
\hline Elliot, A.J & 1 & 32 \\
\hline Alvarez, M. & 1 & 29 \\
\hline Ames, C. & 1 & 28 \\
\hline Boud D. & 1 & 26 \\
\hline Van-Der-Vleuten, C. & 1 & 24 \\
\hline Álvarez Rojo, V; Schmidt, H & 2 & 22 \\
\hline Boekartes, M & 1 & 21 \\
\hline Biggs, J. B; Goleman, D.; Lázaro Martínez, A.; Repetto, E.; Wolfhagen, I. & 4 & 20 \\
\hline Chi, M.; De-Grave, W. & 2 & 19 \\
\hline Brown, A. & 1 & 18 \\
\hline Brown, J.; Extremera Pacheco, N & 2 & 17 \\
\hline Álvarez-Pérez, P.; Echevarría-Samanes, B. & 2 & 16 \\
\hline Álvarez González, M.; Fernández-Berrocal, B. L.; García, M. P.; Gardner, H. & 4 & 15 \\
\hline
\end{tabular}


Tabla 3: Continuación.

\begin{tabular}{|l|c|c|}
\hline Autores & $\mathrm{n}$ & $\mathrm{f}$ \\
\hline Azevedo, R. & 1 & 14 \\
\hline Caruso, D. R.; Cohen, J.; Creswell, J.; W Dweck, C. S & 4 & 13 \\
\hline Álvarez, L.; Bruner, J. S.; Kauchak, D.; Sobrado Fernández, L. & 4 & 12 \\
\hline $\begin{array}{l}\text { Archer, J.; Caprara, G.; Collins A.; De Miguel Díaz, M.; Fernández-Zabala, A.; } \\
\text { García, F.J.; González-Pineda,J.A.; Rodríguez Espinar, S. }\end{array}$ & 8 & 11 \\
\hline $\begin{array}{l}\text { Alonso, J.; Azer, S.; Bolivar Botía, A.; Brown, S. D.; Coll, C.; Dörnyei, Z.; } \\
\text { González, M. C.; Moust, J.; Pintrich, P. R }\end{array}$ & 9 & 10 \\
\hline- & 18 & 9 \\
\hline- & 18 & 8 \\
\hline- & 27 & 7 \\
\hline- & 51 & 6 \\
\hline- & 62 & 5 \\
\hline- & 121 & 4 \\
\hline- & 291 & 3 \\
\hline- & 610 & 2 \\
\hline- & 23994 & 1 \\
\hline Total & 25245 & \\
\hline
\end{tabular}

Tabla 4: Orden de autoría y género por periodos diacrónicos

\begin{tabular}{|c|c|c|c|c|c|}
\hline \multirow{2}{*}{ Orden autoría } & \multirow{2}{*}{$\begin{array}{l}\text { Género } \\
\text { autor/a }\end{array}$} & \multicolumn{4}{|c|}{ Periodo de publicación y (f) } \\
\hline & & $1988 / 1998$ & $1999 / 2008$ & $2009 / 2019$ & Total \\
\hline \multirow{2}{*}{$1^{0}$} & Hombre & 28 & 172 & 231 & 431 \\
\hline & Mujer & 25 & 169 & 394 & 588 \\
\hline \multirow{2}{*}{$2^{\circ}$} & Hombre & 13 & 91 & 198 & 302 \\
\hline & Mujer & 17 & 103 & 256 & 376 \\
\hline \multirow{2}{*}{$3^{\circ}$} & Hombre & 4 & 38 & 129 & 171 \\
\hline & Mujer & 8 & 69 & 153 & 230 \\
\hline \multirow{2}{*}{$4^{\circ}$} & Hombre & 1 & 21 & 53 & 75 \\
\hline & Mujer & 1 & 25 & 64 & 90 \\
\hline \multirow{2}{*}{$5^{0}$} & Hombre & 3 & 7 & 18 & 28 \\
\hline & Mujer & 1 & 10 & 31 & 42 \\
\hline \multirow{2}{*}{$6^{\circ}$} & Hombre & - & 7 & 11 & 18 \\
\hline & Mujer & 1 & 2 & 7 & 10 \\
\hline \multirow{2}{*}{$7^{0}$} & Hombre & - & 2 & 4 & 6 \\
\hline & Mujer & - & 3 & 3 & 6 \\
\hline \multirow{2}{*}{$8^{\circ}$} & Hombre & - & 1 & 2 & 3 \\
\hline & Mujer & - & 2 & 2 & 4 \\
\hline \multirow{2}{*}{ TOTAL } & Hombre & 49 & 339 & 646 & 1034 \\
\hline & Mujer & 53 & 383 & 910 & 1346 \\
\hline
\end{tabular}




\section{Productividad institucional}

El análisis de la productividad institucional se realiza por la filiación de los autores y en el caso de autoría múltiple por la de los autores principales de los trabajos, entendiendo éstos como los que firman la publicación en primer lugar. $Y$ se analiza el número de aportaciones con relación al número de instituciones que las aportan, para poder calcular el índice de transitoriedad y establecer el ajuste al modelo matemático de la distribución de Lotka, como se muestra en la tabla 5. En la que se muestran tres periodos temporales que se ajustan a una productividad en la que la mayor parte de las aportaciones se realizan desde unas pocas universidades. Es decir, la mayoría realizan aportaciones de forma ocasional, ajustándose a las distribuciones de Lotka y Price. Las instituciones con una sola aportación son del 80\%, 69,53\% y 79,12\%, lo que sitúa este nivel de productividad en los valores del índice de transitoriedad establecido ya por Spinak (1996).

En el análisis de la productividad institucional nominal muestra las instituciones más productivas, por periodos temporales, para constatar cambios diacrónicos en el ranking de productividad y su ajuste a la ley de distribución productiva propuesta por Bradford en la tabla 6 . Y el núcleo de mayor productividad en la primera década considerada lo constituyen la U. Complutense de Madrid y MEC, seguido por cinco universidades. Y para igualar su productividad habría que considerar la producción ocasional conjunta de 24 instituciones. Estaos datos muestran además de la variación de las instituciones más productivas, la relevancia de su producción nuclear o especialistas temáticos.

Tabla 5: Aportaciones por instituciones, ajuste a la Ley de Bradford e índice de transitoriedad

\begin{tabular}{|c|c|c|c|c|}
\hline Periodo & № aportaciones & № universidades & $\%$ & I.T. \\
\hline \multirow[t]{6}{*}{ 1988-1998 } & $4-3$ & 1 & 2,38 & 3,33 \\
\hline & 2 & 5 & 11,90 & 16,67 \\
\hline & 1 & 24 & 57,14 & 80,00 \\
\hline & Otras instituciones no universitarias & - & - & - \\
\hline & No indican la filiación institucional & 12 & 28,57 & - \\
\hline & TOTAL & 42 & 100,00 & 100,00 \\
\hline \multirow[t]{11}{*}{$1999-2008$} & $18-12-9-8$ & 1 & 0,48 & 0,78 \\
\hline & 7 & 4 & 1,91 & 3,13 \\
\hline & 6 & 2 & 0,96 & 1,56 \\
\hline & 5 & 4 & 1,91 & 3,13 \\
\hline & 4 & 1 & 0,48 & 0,78 \\
\hline & 3 & 9 & 4,31 & 7,03 \\
\hline & 2 & 18 & 8,61 & 14,06 \\
\hline & 1 & 89 & 42,58 & 69,53 \\
\hline & Otras instituciones no universitarias & 18 & 8,61 & - \\
\hline & No indican la filiación institucional & 63 & 30,14 & - \\
\hline & TOTAL & 209 & 100,00 & 100,00 \\
\hline \multirow[t]{13}{*}{$2009-2019$} & 24.23.18.15-14-12-11 & 1 & 0,26 & 0,34 \\
\hline & 10 & 2 & 0,52 & 0,67 \\
\hline & $9-8$ & 1 & 0,26 & 0,34 \\
\hline & 7 & 2 & 0,52 & 0,67 \\
\hline & 6 & 5 & 1,29 & 1,68 \\
\hline & 5 & 3 & 0,78 & 1,01 \\
\hline & 4 & 6 & 1,55 & 2,02 \\
\hline & 3 & 14 & 3,62 & 4,71 \\
\hline & 2 & 28 & 7,24 & 9,43 \\
\hline & 1 & 235 & 60,72 & 79,12 \\
\hline & Otras instituciones no universitarias & 15 & 3,88 & - \\
\hline & No indican la filiación institucional & 75 & 19,38 & - \\
\hline & Total & 387 & 100,00 & 100,00 \\
\hline
\end{tabular}


Tabla 6: Productividad institucional nominal

\begin{tabular}{|c|c|c|c|}
\hline Periodo & Aportaciones & Número & Universidad \\
\hline \multirow[t]{3}{*}{ 1988-1998 } & $4+3$ & 2 & U. Complutense de Madrid, Ministerio de Educación y Ciencia \\
\hline & 2 & 5 & $\begin{array}{l}\text { U. de Indiana, U. Islas Baleares, U. de Sevilla, U. País Vasco } \\
\text { y U. Kansas }\end{array}$ \\
\hline & 1 & \multicolumn{2}{|c|}{24 instituciones } \\
\hline \multirow[t]{4}{*}{ 1999-2008 } & 18 & 1 & U. Barcelona \\
\hline & $12+9$ & 2 & U. Granada y U. Autónoma de Madrid \\
\hline & $8+7+6$ & 7 & $\begin{array}{l}\text { U. Sevilla, U. León, U. Murcia, U. Santiago de Compostela, } \\
\text { U. País Vasco, U. Complutense de Madrid y U. Salamanca }\end{array}$ \\
\hline & $5+4+3+2+1$ & \multicolumn{2}{|c|}{121 instituciones } \\
\hline \multirow[t]{4}{*}{ 2009-2019 } & $24+23$ & 2 & $\begin{array}{l}\text { Universidad Nacional de Educación a Distancia (UNED) } \\
\text { U. Granada }\end{array}$ \\
\hline & $18+15+14$ & 3 & U. Barcelona, U. Jaén y U. Sevilla \\
\hline & $12+11+10+9$ & 5 & $\begin{array}{l}\text { U. Murcia, U. Complutense de Madrid, U. Córdoba, U. La } \\
\text { Laguna y U. Valencia. }\end{array}$ \\
\hline & $8+7+6+5+4+3+2+1+$ & \multicolumn{2}{|c|}{293 instituciones } \\
\hline
\end{tabular}

\section{Contenidos temáticos de investigación}

Los contenidos temáticos de investigación se analizan partir del estudio de la longitud de los títulos el análisis cualitativo de las palabras que constituyen dicho título y de las palabras clave que los definen mediante NudistVivo. En primer lugar, los títulos muestran un aumento medio de 5,2 palabras por título de la primera a la segunda década considerada y un descenso de 0,51 en la última. Esto supone la estabilización de la longitud, como se muestra en la tabla 7 , y puede interpretarse como una especialización de la temática de la orientación y tutorización en aspectos concretos. El análisis de las palabras contenidas en los títulos muestra un total de palabras distintas que los componen de 1694 con una frecuencia total de 8233 repeticiones. Siendo las palabras más usuales, eliminando los artículos, preposiciones y palabras similares, las que se muestran en la tabla 8. Y considerando la longitud de los títulos como indicador de la complejidad de la materia se muestra un aumento de 5,2 palabras por título de la primera a la segunda década considerada y un descenso de 0,51 en la última, para estabilizarse entorno a una media de 17,84 palabras por título, se asocia a una especificación de las temáticas consideradas.

Tabla 7: Longitud media del número de palabras de los títulos de los trabajos

\begin{tabular}{|l|c|}
\hline Años & Longitud media/década \\
\hline $1988 / 98$ & 13,44 \\
\hline $1999 / 08$ & 18,46 \\
\hline $2009 / 19$ & 17,91 \\
\hline Total & 17,84 \\
\hline
\end{tabular}

Y el análisis de las palabras clave se refiere a una muestra de 7348 palabras clave, registradas en la tabla 9 , en la que se realiza una categorización temática de las palabras clave, que permita una mejor comprensión analítica y comparación diacrónica. Las palabras generales sobre el campo de conocimiento, tales como orientación, tutor, tutoría y aprendizaje, no sufren variaciones en los periodos diacrónicos considerado, siendo siempre las más frecuentemente empleadas como palabras clave de las investigaciones a las que se refieren. Sin embargo, en las siguientes palabras en frecuencia si aparecen algunas diferencias que permiten constatar las variaciones temáticas. Así en el primer periodo aparecen palabras como educación compensatoria, alumno, evaluación y evaluación continua. En el segundo periodo, aparecen términos referidos al desarrollo personal como autoimagen, y adecuaciones de los procesos generales de aprendizaje con términos como auto-aprendizaje, interpretación o estilo de aprendizaje. Y en el último periodo destacan términos de atención ala diversidad e inclusión, aprendizajes activos, participativos o tutorización por pares.

En cuanto a las palabras que componen estos títulos, las más usuales han sido "orientación, tutoría o tutorial"; aunque en la última década hay un ajuste a palabras con un carácter más aplicado en cuanto a las consecuencias de la disciplina con "educación, educativa y enseñanza". Aunque lo relevante consiste en la incorporación en esta última década de términos como "estrategias, formación, inclusión, social, desarrollo y estudiantes" que delimitan una disciplina con un carácter mucho más aplicado a la consecución de metas específicas que representan dichas palabras. Esta especificación también se puede apreciar en el análisis de las palabras clave que han pasado de aspectos generales como "orientación pedagógica, psicopedagógica y 
orientador", a considerar "orientación personal y orientación del currículo" y finalmente incluir como más usuales "orientación personal, académica y profesional". Esto supone un nuevo foco de interés de la investigación educativa y la orientación no sólo ajustada a aspectos académicos y curriculares tradicionales (Abeysekera y Dawson, 2015).

Tabla 8: Palabras usuales empleadas en los títulos

\begin{tabular}{|l|c|c|c|c|c|}
\hline Palabras del título & $f$ & $\%$ & $1960-1998$ & $1999-2008$ & $2099-2019$ \\
\hline Educación + educativa + enseñanza & 505 & 33,98 & 34 & 92 & 379 \\
\hline Aprendizaje & 329 & 22,14 & 5 & 71 & 253 \\
\hline Orientación + tutoría + tutorial & 355 & 23,89 & 44 & 154 & 157 \\
\hline Estrategias & 76 & 5,11 & 0 & 10 & 66 \\
\hline Formación & 51 & 3,43 & 7 & 16 & 28 \\
\hline Inclusión & 44 & 2,96 & 0 & 1 & 43 \\
\hline Social & 43 & 2,89 & 2 & 9 & 32 \\
\hline Desarrollo & 42 & 2,83 & 7 & 16 & 19 \\
\hline Estudiantes & 41 & 2,76 & 0 & 7 & 34 \\
\hline Total & 1486 & 100 & 99 & 376 & 1011 \\
\hline
\end{tabular}

Tabla 9: Palabras clave empleadas por intervalos diacrónicos

\begin{tabular}{|c|c|c|c|}
\hline Periodo & f & $n$ & Palabras clave (categorizadas) \\
\hline \multirow[t]{10}{*}{ 1988-1998 } & 35 & 1 & Orientación pedagógica, psicopedagógica, orientador \\
\hline & 10 & 1 & Tutor, tutoría, roles \\
\hline & 8 & 3 & Profesor, tutor/ Estudios/Programa, proyecto de curso, de enseñanza, de \\
\hline & 7 & 5 & $\begin{array}{l}\text { Educación global/ educación/ educación compensatoria } \\
\text { / educación de la primera infancia/ educación moral }\end{array}$ \\
\hline & 6 & 1 & Enseñanza Secundaria \\
\hline & 4 & 3 & Alumno/ enseñanza primaria/ Evaluación, evaluación continua, evaluación escolar/ \\
\hline & 3 & 5 & - \\
\hline & 2 & 19 & - \\
\hline & 1 & 54 & - \\
\hline & - & 134 & NS/NR \\
\hline \multirow[t]{15}{*}{ 1999-2008 } & 111 & 1 & Orientación, orientador, orientación personal, del currículo \\
\hline & 45 & 1 & Tutor, tutoría, roles \\
\hline & 41 & 1 & Educación, educación crítica, emocional, inclusiva, familiar, moral \\
\hline & 26 & 1 & Auto concepto, auto estima, auto imagen, física, académica \\
\hline & 17 & 1 & Aprendizaje \\
\hline & 16 & 1 & Aprendizaje basado en problemas \\
\hline & 15 & 1 & Enseñanza \\
\hline & 14 & 1 & Desarrollo, cognitivo, personal, para la carrera \\
\hline & 12 & 2 & Fluidez de interpretación/ estudiantes, actitudes, interacción \\
\hline & 11 & 2 & $\begin{array}{l}\text { Aprendizaje, aprendizaje autorregulado, cooperativo de lenguas, social/ Programa de } \\
\text { formación }\end{array}$ \\
\hline & 10 & 3 & $\begin{array}{l}\text { Estilo de aprendizaje y estrategias de aprendizaje/ Evaluación/ Inteligencia, inteligencia } \\
\text { emocional, inteligencias múltiples }\end{array}$ \\
\hline & 8 & 2 & $\begin{array}{l}\text { Competencias, transversales, profesionales, sociales/ Intervención, en clase, } \\
\text { pedagógica, cognitiva }\end{array}$ \\
\hline & 7 & 3 & Acción tutorial/ Meta cognitivo, meta cognición/ Motivación \\
\hline & 6 & 8 & $\begin{array}{l}\text { Aprendizaje y tutorización entre iguales/ Cooperación/ Discapacidad/ Enseñanza } \\
\text { secundaria/ Investigación/ Profesor }\end{array}$ \\
\hline & 5 & 5 & Comunicación/ diagnóstico/ matemáticas/ rendimiento/ universidad \\
\hline
\end{tabular}


Tabla 9 (Continuación)






\section{Tipología y lengua de la publicación}

El análisis de la publicación considera indicadores como la tipología de publicación y lengua empleada, el estudio nominal de las fuentes, las referencias citadas y la citación recibida. Comenzando por el análisis de la tipología de publicación y lengua empleada se muestra un registro (ver tabla 10) que constata de forma global una mayoría de publicaciones en tipología artículo en revista científica, publicado en español. Y en segundo lugar este tipo de fuente, pero publicada en inglés. Otras tipologías de publicaciones no se suelen registrar en las bases de datos, por lo que arrojan valores escasos en frecuencia y porcentaje.

Aunque el idioma inglés no es el idioma más hablado del mundo sin embargo es considerado uno de los más importantes y reconocido como lengua internacional en la producción científica, en este caso la lengua más empleada es el español y el país de edición España. Este aspecto puede indicar que la orientación, tutoría y acción tutorial viene siendo un tópico recurrente en la investigación educativa de nuestro país, en el que Scimago Journal \& Country Rank sitúa como la sexta en el ranking de publicaciones científicas con un total de 14.920 documentos solo en educación, siendo el séptimo que más citas recibas de un total de 209 países repartidos por todo el mundo. Sin embargo, aunque los trabajos más citados son igualmente los artículos en revista científica, los trabajos más citados hacen referencia a investigaciones publicadas en inglés, lo que supone una dependencia hacia las fuentes más citadas (Lowry, Romans y Curtis, 2004).

Tabla 10: Tipología de publicación y lengua empleada

\begin{tabular}{|c|c|c|c|c|c|c|c|c|c|}
\hline \multirow[b]{2}{*}{ Tipo de fuente } & \multirow[b]{2}{*}{ Idioma } & \multicolumn{2}{|c|}{ 1988-1998 } & \multicolumn{2}{|c|}{$1999-2008$} & \multicolumn{2}{|c|}{$2009-2019$} & \multicolumn{2}{|c|}{ TOTAL } \\
\hline & & $f$ & $\%$ & $f$ & $\%$ & $f$ & $\%$ & $f$ & $\%$ \\
\hline \multirow{3}{*}{$\begin{array}{l}\text { Artículo en revista } \\
\text { científica (ARC) }\end{array}$} & Inglés & 21 & 30,43 & 117 & 33,72 & 226 & 35,42 & 173,42 & 33,61 \\
\hline & Español & 38 & 55,07 & 183 & 52,74 & 316 & 49,53 & 270,53 & 52,43 \\
\hline & Otros & - & - & - & - & 4 & 0,63 & 0,63 & 0,12 \\
\hline \multirow{3}{*}{$\begin{array}{l}\text { Artículo en revista } \\
\text { general (ARG) }\end{array}$} & Inglés & - & - & - & - & 1 & 0,16 & 0,16 & 0,03 \\
\hline & Español & - & - & - & - & - & - & - & - \\
\hline & Otros & - & - & - & - & - & - & - & - \\
\hline \multirow{3}{*}{ Tesis doctoral (TDC) } & Inglés & - & - & 11 & 3,17 & 39 & 6,11 & 17,11 & 3,32 \\
\hline & Español & 1 & 1,45 & - & - & 11 & 1,72 & 2,72 & 0,53 \\
\hline & Otros & - & - & - & - & - & - & - & - \\
\hline \multirow{3}{*}{$\begin{array}{l}\text { TFM, tesina, TFG o } \\
\text { similares (TFE) }\end{array}$} & Inglés & - & - & - & - & - & - & - & - \\
\hline & Español & 1 & 1,45 & 2 & 0,58 & 3 & 0,47 & 3,47 & 0,67 \\
\hline & Otros & - & - & - & - & - & - & - & - \\
\hline \multirow{3}{*}{ Libro completo (LBR) } & Inglés & - & - & - & - & - & - & - & - \\
\hline & Español & - & - & 2 & 0,58 & 1 & 0,16 & 2,16 & 0,42 \\
\hline & Otros & - & - & - & - & - & - & 0,00 & 0,00 \\
\hline \multirow{3}{*}{ Libro capítulo (LBC) } & Inglés & - & - & - & - & - & - & - & - \\
\hline & Español & 1 & 1,45 & - & - & - & - & 1,00 & 0,19 \\
\hline & Otros & - & - & - & - & - & - & - & - \\
\hline \multirow{4}{*}{ Otras fuentes (OTR) } & Inglés & - & - & 10 & 2,88 & 24 & 3,76 & 13,76 & 2,67 \\
\hline & Español & 7 & 10,14 & 21 & 6,05 & 13 & 2,04 & 30,04 & 5,82 \\
\hline & Otros & - & - & 1 & 0,29 & - & - & 1,00 & 0,19 \\
\hline & TotaL & 69 & 100 & 347 & 100 & 638 & 100 & 516,00 & 100 \\
\hline
\end{tabular}

\section{Fuentes de publicación}

Las fuentes de publicación agrupadas por grupos de productividad considerados en la Ley de Bradford, ver tabla 11, en los tres periodos temporales considerados muestran una producción temática ocasional. Algunas fuentes destacan por su pervivencia productiva como la Revista de Educación o por su alta productividad temática como REOP.

Las fuentes de publicación tienen un origen o país de publicación que hace referencia a su registro legal. En este estudio se considera como ámbito geográfico de publicación y tradición escolástica que representan. Es 
cierto que por razones experimentales pudiera haber un cierto sesgo positivo hacia la productividad española, pero la consideración en la discusión de datos más que centrarse en el país específico, hace referencia a los cambios cronológicos apreciados. Pero el análisis por áreas geográficas muestra la preponderancia de la zona europea y la nueva incorporación científica del área Asia-Pacífico y Suramérica en la última década (Ver tabla 12). Sin embargo, la productividad de países tradicionalmente productores como España, EEUU o Reino Unido permanece con cierta constancia, parece haber un mayor número de países productores y una descentralización geográfica.

Tabla 11: Productividad por fuentes de publicación

\begin{tabular}{|c|c|c|c|}
\hline Periodo & Aportaciones por fuente & $\begin{array}{l}\text { Número } \\
\text { de fuentes }\end{array}$ & Nombre de la fuente \\
\hline \multirow[t]{4}{*}{$1988-1998$} & $9+8$ & 2 & Vida Escolar, Revista de Educación \\
\hline & $5+5+3+3$ & 4 & $\begin{array}{l}\text { Remedial and Special Education: RASE, Revista } \\
\text { Complutense de Educación, Education \& Treatment of } \\
\text { Children, The Journal of Higher Education }\end{array}$ \\
\hline & 1 ó 2 & \multicolumn{2}{|l|}{26} \\
\hline & NS/NR & \multicolumn{2}{|l|}{9} \\
\hline \multirow[t]{7}{*}{$1999-2008$} & 29 & 1 & Revista Española de Orientación y Psciopedagogía \\
\hline & $12+12+10$ & 3 & $\begin{array}{l}\text { Educación XXI : Revista de la Facultad de Educación, } \\
\text { Journal of Learning Disabilities, Remedial and Special } \\
\text { Education: RASE }\end{array}$ \\
\hline & $10+10+8+8$ & 4 & $\begin{array}{l}\text { Revista de Educación, RIE: Revista de investigación } \\
\text { educativa, Revista de Psicodidáctica, Tendencias } \\
\text { pedagógicas }\end{array}$ \\
\hline & $7+7+7+6+5$ & 5 & \\
\hline & $4+4+4+4+3+3+3+3+3+3$ & \multicolumn{2}{|l|}{10} \\
\hline & 2 ó 1 & \multicolumn{2}{|l|}{134} \\
\hline & NS/NR & \multicolumn{2}{|l|}{20} \\
\hline \multirow[t]{6}{*}{$2009-2019$} & 75 & 1 & Revista Española de Orientación y Psicopedagogía \\
\hline & $19+13+10+10+8+8$ & 6 & $\begin{array}{l}\text { Educación XXI: Revista de la Facultad de Educación, } \\
\text { Educación Siglo XXI, Revista de Educación, Prisma } \\
\text { Social, Revista Complutense de Educación, Enseñanza } \\
\text { \& Teaching }\end{array}$ \\
\hline & $\begin{array}{l}7+7+7+6+6+5+5+5+5+4+4+4+4 \\
+4+4+4+4\end{array}$ & \multicolumn{2}{|c|}{$\begin{array}{ll}17 & \text { British Journal of Guidance and Counselling } \\
7 & \\
\end{array}$} \\
\hline & 3 & \multicolumn{2}{|l|}{13} \\
\hline & 2 ó 1 & \multicolumn{2}{|l|}{359} \\
\hline & NS/NR & \multicolumn{2}{|l|}{18} \\
\hline
\end{tabular}

Tabla 12: Productividad por áreas geográficas.

\begin{tabular}{|c|c|c|c|c|}
\hline & 1988-1998 & 1999-2008 & $2009-2019$ & TOTAL \\
\hline Europa & 38 & 201 & 405 & 644 \\
\hline Norteamérica & 18 & 74 & 118 & 210 \\
\hline Suramérica & 0 & 0 & 31 & 31 \\
\hline Asia-Pacífico & 0 & 17 & 56 & 73 \\
\hline África & 0 & 0 & 4 & 4 \\
\hline Otros & 0 & 2 & 21 & 23 \\
\hline NS/NR & 0 & 53 & 3 & 0 \\
\hline Total & 56 & 347 & 638 & 1041 \\
\hline
\end{tabular}

Citación en los trabajos revisados

Las citas realizadas en cada trabajo se cuantifican por el número de referencias registradas y se modula por el tipo de fuente empleada para publicar, empleando la misma notación de la tipología de fuentes que la tabla 
13. Siendo la tipología de trabajo más referenciado son los artículos de revistas. Y el índice de referencias medias por trabajo es para los artículos en revistas entorno a 36 y para las tesis doctorales $>150$. Siendo ambos índices usuales en Ciencias Sociales.

Las citas recibidas se calculan empleando los registros de Google Schoolar y se registran por la lengua de publicación, en la tabla 14, en la que $(\mathrm{N})$ hace referencia al número trabajos publicados e (IC) al índice de citación o número de citas medio recibido por trabajo. En todos los periodos se constata una mayor citación a trabajos en lengua inglesa y un mayor índice de citación por trabajos producidos en dicha lengua, aspectos ya considerados por Belikov y Belikov (2015).

\section{Indicadores metodológicos de los estudios revisados}

Los indicadores metodológicos empleados en esta revisión, como se muestra en la tabla 16, hacen referencia a la metodología empleada y los instrumentos de recogida de información empleados en los estudios revisados. La evolución del uso de metodologías específicas no muestra un patrón temporal claro. Sin embargo, resulta significativa la falta de especificidad declarativa de la metodología empleada $(27,69 \%)$ o su catalogación como metodología cuantitativa de forma general $(28,92 \%)$.

Tabla 13: Tipología de los trabajos citados

\begin{tabular}{|l|l|l|l|l|l|l|l|l|l|l|}
\hline & \multicolumn{2}{l}{$1988-1998$} & \multicolumn{2}{l}{$1999-2008$} & \multicolumn{2}{l|}{$2009-2019$} & \multicolumn{2}{l|}{ TOTAL } & N & I.R. \\
\hline & $\mathrm{f}$ & $\%$ & $\mathrm{f}$ & $\%$ & $\mathrm{f}$ & $\%$ & $\mathrm{f}$ & $\%$ & & \\
\hline ARC & 1849 & 72,62 & 11027 & 86,84 & 19770 & 69,33 & 32646 & 228,79 & 902 & 36,19 \\
\hline ARG & - & - & - & - & 46 & 0,16 & 46 & 0,16 & 1 & 46,00 \\
\hline TDC & 285 & 11,19 & 1551 & 12,21 & 7532 & 26,41 & 9368 & 49,82 & 62 & 151,10 \\
\hline TFE & 47 & 1,85 & 120 & 0,95 & 252 & 0,88 & 419 & 3,67 & 6 & 69,83 \\
\hline LBR & - & - & - & - & - & - & - & - & - & - \\
\hline LBC & - & - & - & - & - & - & - & - & - & - \\
\hline OTR & 365 & 14,34 & - & - & 916 & 3,21 & 1281 & 17,55 & 76 & 16,86 \\
\hline
\end{tabular}

Tabla 14: Lengua de los trabajos citados

\begin{tabular}{|l|r|c|c|c|r|r|r|r|r|r|}
\hline & \multicolumn{2}{|c|}{$1988-1998$} & \multicolumn{2}{c|}{$1999-2008$} & \multicolumn{2}{c|}{$2009-2019$} & \multicolumn{2}{|c|}{ TOTAL } & N & I.C. \\
\hline & \multicolumn{1}{c|}{$\mathrm{f}$} & \multicolumn{1}{c|}{$\%$} & $\mathrm{f}$ & \multicolumn{1}{c|}{$\%$} & $\mathrm{f}$ & \multicolumn{1}{c|}{$\%$} & $\mathrm{f}$ & \multicolumn{1}{c|}{$\%$} & & \\
\hline Inglés & 1338 & 82,09 & 9729 & 77,23 & 3399 & 63,01 & 14466 & 73,72 & 450 & 32,15 \\
\hline Español & 292 & 17,91 & 2869 & 22,77 & 1987 & 36,84 & 5148 & 26,24 & 596 & 8,64 \\
\hline Otros & - & - & - & - & 8 & 0,15 & 8 & 0,04 & 5 & 1,60 \\
\hline Total & 1630 & 100 & 12598 & 100 & 5394 & 100 & 19622 & 100 & 1051 & \\
\hline
\end{tabular}

Tabla 15: Metodología empleada en los estudios revisados

\begin{tabular}{|c|c|c|c|c|c|c|c|c|}
\hline \multirow[b]{2}{*}{ Metodología } & \multicolumn{2}{|c|}{ 1988-1998 } & \multicolumn{2}{|c|}{ 1999-2008 } & \multicolumn{2}{|c|}{$2009-2019$} & \multicolumn{2}{|c|}{ TOTAL } \\
\hline & $f$ & $\%$ & $f$ & $\%$ & $f$ & $\%$ & $f$ & $\%$ \\
\hline C. $\sin$ especificar & 1 & 7,14 & 18 & 32,73 & 285 & 29,02 & 304 & 28,92 \\
\hline C. pre-experimental & - & - & - & - & 1 & 0,10 & 1 & 0,10 \\
\hline C. cusi-experimental & - & - & - & - & 29 & 2,95 & 29 & 2,76 \\
\hline C .experimental & - & - & 3 & 5,45 & 71 & 7,23 & 74 & 7,04 \\
\hline Q. sin especificar & - & - & 7 & 12,73 & 175 & 17,82 & 182 & 17,32 \\
\hline $\begin{array}{l}\text { Q. }{ }^{1} \text { Historias de vida, métodos narrativos, } \\
\text { análisis del discurso, etc. }\end{array}$ & - & - & 0 & 0,00 & 41 & 4,18 & 41 & 3,90 \\
\hline $\begin{array}{l}\text { Q. }{ }^{2} \text { Estudios de casos únicos, análisis del } \\
\text { desempeño, etc. }\end{array}$ & - & - & 3 & 5,45 & 29 & 2,95 & 32 & 3,04 \\
\hline $\mathrm{X}$ métodos mixtos & - & - & 2 & 3,64 & 95 & 9,67 & 97 & 9,23 \\
\hline No especifica & 13 & 92,86 & 22 & 40,00 & 256 & 26,07 & 291 & 27,69 \\
\hline Total & 14 & 100 & 55 & 100 & 982 & 100 & 1051 & 100 \\
\hline
\end{tabular}


En cuanto a los aspectos metodológicos de las investigaciones publicadas, que se especifican en la tabla 15 , como afirma Bisquerra (2009) el conocimiento científico en la educación es una mezcla de conocimiento teórico y acción práctica por lo que su método de investigación debe adecuarse a la naturaleza de su investigación. Sin embargo resulta significativa la falta de especificidad declarativa de la metodología empleada o en el mejor de los casos su catalogación como metodología cuantitativa (C) de forma general $(28,92)$, cualitativa $(Q) \sin$ especificar $(17,32 \%)$ o directamente sin especificación alguna (27,69\%). Lo que sin duda puede representar una cierta laxitud metodológica (Chang, Chang y Tseng, 2010).

La tipología de fuentes e instrumentos de recogida de información, registrados en la tabla 16, muestra que las fuentes indirectas poseen cierta estabilidad temporal, atendiendo a los intervalos temporales considerados en su uso, siendo el más empleado en todos ellos la entrevista. $Y$ en las fuentes directas, sigue habiendo un amplio predominio prototípico en Ciencias Sociales, del cuestionario. Destacando todavía el escaso empleo de software para la recogida de informaciones. Los instrumentos más empleados siguen siendo los cuestionarios, considerado un instrumento clásico en las ciencias sociales para la obtención y registro de datos ya que debido a su versatilidad permite abarcar aspectos cuantitativos y cualitativos. Aunque ello suponga un detrimento del uso de otros instrumentos que pudieran ser más ajustados (Fernández-Cano y Expósito, 2004).

Con las limitaciones propias de los estudios cienciométricos, como planteaban Archambault y Larivière (2010), puede afirmarse que la orientación, tutorización y acción tutorial es una disciplina productivamente madura y de relevancia en nuestro contexto geográfico, con la incorporación de aspectos referidos al desarrollo de la personalidad y el aprendizaje, más allá de los tradicionalmente focalizados en lo académico y curricular. $Y$ aunque existen autores y fuentes nucleares, como ocurre en otras disciplinas del área de ciencias sociales, la producción ocasional tiene altos índices. De igual forma, aunque se poseen altas tasas de producción y de edición de trabajos, las fuentes de citación siguen haciendo referencia a fuentes anglosajonas, lo que puede considerarse una practica tradicional o una debilidad epistemológica estructural

Tabla 16: Fuentes e instrumentos para la recogida de información empleados

\begin{tabular}{|c|c|c|c|c|c|c|c|c|}
\hline \multirow[t]{2}{*}{ Tipo } & \multicolumn{2}{|c|}{ 1988-1998 } & \multicolumn{2}{|c|}{ 1999-2008 } & \multicolumn{2}{|c|}{$2009-2019$} & \multicolumn{2}{|c|}{ TOTAL } \\
\hline & $f$ & $\%$ & $f$ & $\%$ & $f$ & $\%$ & $f$ & $\%$ \\
\hline \multicolumn{9}{|l|}{ Fuentes indirectas } \\
\hline Documentos & 6 & 10,00 & 33 & 10,38 & 74 & 9,24 & 113 & 9,58 \\
\hline Notas de campo & 0 & 0,00 & 13 & 4,09 & 48 & 5,99 & 61 & 5,17 \\
\hline Observación & 12 & 20,00 & 37 & 11,64 & 79 & 9,86 & 128 & 10,86 \\
\hline Entrevistas & 8 & 13,33 & 37 & 11,64 & 135 & 16,85 & 180 & 15,27 \\
\hline Grupos de discusión & 1 & 1,67 & 10 & 3,14 & 48 & 5,99 & 59 & 5,00 \\
\hline \multicolumn{9}{|l|}{ Fuentes directas } \\
\hline Escalas & 11 & 18,33 & 37 & 11,64 & 56 & 6,99 & 104 & 8,82 \\
\hline Cuestionarios & 9 & 15,00 & 90 & 28,30 & 261 & 32,58 & 360 & 30,53 \\
\hline Test & 6 & 10,00 & 50 & 15,72 & 78 & 9,74 & 134 & 11,37 \\
\hline Inventarios & 0 & 0,00 & 1 & 0,31 & 0 & 0,00 & 1 & 0,08 \\
\hline Pruebas a /hoc & 2 & 3,33 & 1 & 0,31 & 14 & 1,75 & 17 & 1,44 \\
\hline Programas y app & 5 & 8,33 & 9 & 2,83 & 7 & 0,87 & 21 & 1,78 \\
\hline NS/NC & 0 & 0,00 & 0 & 0,00 & 1 & 0,12 & 1 & 0,08 \\
\hline Total & 60 & 100 & 318 & 100 & 801 & 100 & 1179 & 100 \\
\hline
\end{tabular}

\section{CONCLUSIONES}

De acuerdo con el trabajo presentado, a las discusiones sobre las diversas propiedades estudiadas, y a los resultados obtenidos en cada caso, se pueden plantear las siguientes conclusiones principales ajustadas a los objetivos planteados:

1. La productividad diacrónica en el tópico de investigación sobre orientación, tutorización y acción tutorial muestra un incremento creciente ajustado a una curva logística y un aumento del índice de colaboración, ya registrada por la CNEAI (2017), lo que supone el interés progresivo y cierta madurez productiva, ya recogido en análisis como los de Salgado-Orellana, Ferrada, Puraivan y Parraguez (2018), aunque con necesidades de mejora como se refleja en este estudio. 
2. La autoría nominal muestra un número limitado de autores e instituciones especialistas, con un alto índice de transitoriedad y escasa especialización temática, muy prototípico en las ciencias sociales como afirmaba Spinak (1996). La relevancia educativa y social de la disciplina requiere el establecimiento de medidas que fomenten la especialización. Y pese a los avances en los últimos años en la productividad por género y el mayor número global de mujeres firmantes, no evitan que siga existiendo discriminación de género en los primeros lugares de firma de los trabajos. Este dato confirma la necesidad de políticas científicas activas que consideren estos aspectos, como las que se plantean de forma general por la Comisión Europea (2014).

3. Los contenidos de la investigación sobre orientación, tutorización y acción tutorial se han adaptado desde aspectos disciplinares generales, como concluía en su estudio de revisión López-Gómez (2016) y contenidos académico-curriculares (Ferreira-Villa, Pascual-García y Pol-Asmarats, 2013) a requerimientos para la resolución adaptada de situaciones específicas como la orientación personal, académica sobre el aprendizaje o profesional.

4. La fuente de publicación prioritaria de los informes de investigación sigue siendo la revista científica y la publicación en lengua inglesa, como patrón usual de investigación recogido en trabajos como los de Belikov y Belikov (2015). De igual forma que en el análisis de la productividad personal, existen un limitado número de revistas especializadas y un amplio número de revistas que publican de forma ocasional sobre este tópico.

5. Y de igual forma, la citación media es a 36 trabajos, aunque este dato pueda tener un componente más editorial que científico. Los trabajos más citados son artículos de revistas científicas publicados en lengua inglesa (Belikov y Belikov, 2015).

6. Los aspectos metodológicos se indican en los trabajos publicados de forma general, con escasa especificación de diseños y metodologías, pese a la publicación en revistas especializadas. Con una escasa diversificación de instrumentos de recogida de información que se centran de forma mayoritaria en el uso del cuestionario. Sin embargo este es un elemento que se especifica de forma más clara en estudios cienciométricos de otras disciplinas (Chang, Chang y Tseng, 2010).

De forma general y atendiendo a la finalidad general del estudio, se puede afirmar que la orientación, tutorización y acción tutorial es una disciplina productivamente madura y de relevancia en el ámbito educativo. Pero que requiere de mejoras, como las que se especifican en estas conclusiones, para seguir desarrollándose como disciplina educativa y campo de investigación científica.

Las limitaciones son las propias de los estudios cienciométricos por posibles sesgos en la selección de los descriptores de búsqueda en las bases de datos y la propia selección subjetiva de los registros localizados para su registro como muestra productora de datos. Por ello, se ha planteado un proceso estructurado basado en el consenso entre los investigadores que minimizase estos sesgos. Y la consideración de éstos como elementos de mejora y prospectiva del trabajo, que en algunos indicadores puede automatizarse y potenciarse en sus análisis mediante el empleo de nuevo software como Bibliometrix R.

\section{REFERENCIAS}

Abeysekera, L., y Dawson, P., Motivation and cognitive load in the flipped classroom: Definition, rationale and a call for research. https://doi.org/10.1080/07294360.2014.934336, Higher Education Research \& Development, 34, 1-14 (2015)

Archambault, E., y Larivière, V., The limits of bibliometrics for the analysis of the social sciences and humanities literature: World Social Sciences Report, International Social Science Council, UNESCO Publishing, 251-254 (2010)

Belikov, A. V., y Belikov, V. V., A citation-based, author- and age-normalized, logarithmic index for evaluation of individual researchers independently of publication counts, https://doi.org/10.12688/f1000research.7070.1, Research Open Science, 4, 884-888 (2015)

Bracho, R., Torralbo, M., Maz-Machado, A., y Adamuz, N., Tendencias temáticas de la investigación en educación matemática en España, https://doi.org/10.1590/1980-4415v28n50a04, Bolema, 28 (50), 1077-1094 (2014)

Brooks, T. A., Evidence of complex citer motivations, https://doi.org/10.1002/(SICI)1097-4571(198601)37:1\%3C34::AIDASI5\%3E3.0.CO;2-0, Journal of the American Society for Information Science, 37(1), 34-36 (1986)

Build, R. F., Beltrán, J. M. G., Miedes, A. C., y Martínez, A. M., La orientación educativa en la Revista Española de Orientación y Psicopedagogía. Un estudio diacrónico y sincrónico (1990-2006), https://doi.org/10.4067/S071809342006000200002 , Bordón, 62 (1), 49-59 (2010)

Callon, M., Courtial, J.P. y Penan, H., Cienciometría. El estudio cuantitativo de la actividad científica: De la bibliometría a la vigilancia tecnológica, Tea, $2^{\circledR}$ edición, Gijón, España (1995)

Chang, Y. H., Chang, Ch.Y., y Tseng, Y.H., Trends of Science Education Research: An Automatic Content Analysis, https://doi.org/10.1007/S10956-009-9202-2, Journal of Science Education and Technology, 19, 315-331 (2010)

Expósito, J., Tutorial Action, Síntesis, 1a edición, Madrid, España (2014) 
European Commission., Promoting Gender Equality in Research and Innovation, Horizon 2020, EU Publishing Office, Luxemburg (2014)

Fernández-Cano, A., y Bueno, A., Synthesizing Scientometric Patterns in Spanish Educational Research, https://doi.org/10.1007/S10956-009-9202-210.1007/BF02464783, Scientometrics, 46 (2), 349-367 (1999)

Fernández-Bautista, A., Torralbo, M., y Cano, A. F., Análisis longitudinal de tesis doctorales españolas en educación (1841-2012), doi: 10.7203/relieve.20.2.4479, RELIEVE, 20(2), 1-15 (2014)

Fernández-Cano, A., y Expósito, J., Patrones de citación en la investigación española sobre evaluación de programas educativos (1975-200), https://doi.org/10.1007/S10956-009-9202-210.3989/redc.2001.v24.i3.60, Revista Española de Documentación Científica, 23 (4), 289-305 (2004)

Ferreira-Villa, C., Pascual-García, L., y Pol-Asmarats, C., La producción española en Tesis Doctorales sobre orientación en la Base de Datos TESEO (2001-2012), https://doi.org/10.1007/S10956-009-9202-210.7203/relieve.19.1.2460, RELIEVE, 19 (1), 1-10 (2013)

Leydesdorff, L., The Challenge of Scientometrics: The development, measurement, and self-organization of scientific communications, $1^{\underline{a}}$ edición, Universal Publishers, Florida, USA (2001)

López-Gómez, E., Análisis de las tesis doctorales sobre tutoría: Aproximación bibliometríca y tendencias temáticas, https://doi.org/10.1007/S10956-009-9202-210.5209/rev_RGID.2016.v26.n1.53047, Revista General de Información y Documentación, 26 (1), 147-164 (2016)

Lowry, P. B., Romans, D., y Curtis, A.M., Global Journal Prestige and Supporting Disciplines: A Scientometric Study of Information Systems Journals, Journal of the Association for Information Systems (JAIS), 5 (2), 29-80 (2004)

Ministerio de Educación, Cultura y Deporte (MECD)., Resolución de 23 de noviembre de 2017, de la Comisión Nacional Evaluadora de la Actividad Investigadora, por la que se publican los criterios específicos aprobados para cada uno de los campos de evaluación, Boletín Oficial del Estado, 117067-117080 (2017)

Merton, R. K., Éléments de théorie et de méthode sociologique, $1^{a}$ edición, Marie Mendras, Paris, Francia (1965)

Salgado-Orellana, N., Ferrada, C., Puraivan, E., y Parraguez, R., Un estudio cienciométrico sobre las tesis doctorales españolas en acción tutorial (1980-2016), https://doi.org/10.5209/RGID.62832, Revista General de Información y Documentación, 28 (2), 415-434 (2018)

Sánchez-Ruiz, E., Medios de comunicación y democracia, 1a edición, Editorial Norma, Bogotá, Colombia (2005)

Secretaría de Estado de Universidades, Investigación y Desarrollo., Científicas en cifras, Estadísticas e indicadores de la desigualdad de genero en la formación y profesión científica, Madrid, España (2017).

Spiegel-Rösing, I., y de Solla Price, D., Science, Technology and Society: A Cross-disciplinary Perspective, $1^{\text {a }}$ edición, SAGE, California, USA (1977)

Spinak, E., Diccionario enciclopédico de bibliometría, cienciometría e informetría, $1^{\text {a }}$ eidción, UNESCO, Venezuela (1996)

Van Raan, A. F. J., y Tijssen, R. J. W., Numerical methods for information on aspects of science: Scientometric analysis and mapping in Mapping of Technology: A First Exploration of Knowledge Diffusion Amongst Fields of Technology, Centre for Science and Technology Studies of The University of Leiden, Indiana, USA (1990) 
